\title{
Predictors of Cerebrospinal Fluid Leaks in Endoscopic Surgery for Pituitary Tumors
}

\author{
Mahmoud Saad ${ }^{1, *}$, Mohamed Elkahwagi', Ahmed Musaad², Yasser Khafagy², \\ Ahmed Nageeb Taha ${ }^{1}$ \\ ${ }^{1}$ Department of Neurosurgery, Faculty of Medicine, Mansoura University, Mansoura, Egypt \\ ${ }^{2}$ Department of Otolaryngology, Faculty of Medicine, Mansoura University, Mansoura, Egypt \\ Email address: \\ dr_mhmodsaad@yahoo.com (M. Saad) \\ ${ }^{*}$ Corresponding author
}

\section{To cite this article:}

Mahmoud Saad, Mohamed Elkahwagi, Ahmed Musaad, Yasser Khafagy, Ahmed Nageeb Taha. Predictors of Cerebrospinal Fluid Leaks in Endoscopic Surgery for Pituitary Tumors. International Journal of Neurosurgery. Vol. 3, No. 2, 2019, pp. $26-31$. doi: $10.11648 /$ j.ijn.20190302.13

Received: November 19, 2019; Accepted: December 2, 2019; Published: December 10, 2019

\begin{abstract}
Background: Cerebrospinal fluid (CSF) leak is a serious complication of endoscopic endonasal surgery for pituitary adenoma; leading to a more serious and financial burden of the surgery. Prediction of the occurrence of such sequale and proper plaining for reconstruction can reduce its prevalence and associated morbidities. Purpose: To determine the predictors and risk factors associated with intra/and postoperative cerebrospinal fluid (CSF) leaks in endoscopic endonasal pituitary surgery. Methods: A retrospective study included 150 patients who underwent endoscopic endonasal approach for resection of pituitary adenomas between 2015 and 2018. We analyzed the association between CSF leaks (intra/post-operative) and perioperative variables including patients' demographic data, medical comorbidities, tumor characteristics and operative data (rates of intraoperative leak, reconstructive techniques). Results: 34 patients $(22.6 \%)$ out of the 150 patients had an overall, CSF leak: $26(76.5 \%)$ patients had intraoperative leaks; 5 patients out of them continued to have a postoperative leak. 8 patients (23.5\%) had postoperative leak. Based on multiple logistic regression model analysis; increased body mass index (BMI), associated hydrocephalus, suprasellar extension and recurrent tumors had a significant association with intraoperative CSF leaks, while only BMI and hydrocephalus predicted postoperative CSF leaks. Conclusion: Elevated BMI, suprasellar extension of the tumor, recurrence or previous radiation are all important risk factors that have been proved in this study to have statistically significant association with the incidence of CSF leak. In high risk patients for CSF leakage: adequate reconstruction planis advised to avoid such morbidity.
\end{abstract}

Keywords: CSF, Leak, Endoscopic Endonasal, Pituitary Surgery, Predictors

\section{Introduction}

Endoscopic endonasal skull base surgery has become widely implemented to be the gold standard in surgical treatment of pituitary tumors that have an estimated prevalence of $15 \%$. Endoscopic endonasal approaches (EEA) has superior efficacy compared with microscopic approaches, as it provides enhanced visualization of the surgical field and decrease the incidence rate of postoperative cerebrospinal fluid (CSF) leaks and allow for proper management of such problem if occurred $[1,2,5]$.

Unfortunately, CSF leaks remain one of the most prevalent complications in endoscopic skull base surgery in the literature. Different cohort studies reported rates of CSF leak ranging between $2 \%$ and $64 \%$ [1, 17]. Intraoperative leaks are more common $(14.2 \%-61 \%)$ than postoperative leaks $(1.3 \%-16.7 \%)$, and many patients experienceing postoperative leaks have an observed intraoperative leak [6]. CSF leakage pathogenesis is explained by arachnoid violation during tumor resection. CSF leaks after endoscopic skull base surgery can lead to meningitis, subsequent prolonged morbidity, longer hospitalization time and further financial burden [18].

Identification of preoperative risk factors can guide the 
preoperative planning for intraoperative CSF leakage reconstruction procedure from the start to achieve low morbidity rate especially in patients at increased risk [15].

Many reports in the literature had studied the impact of different risk factors included: patient and tumor variables; age, race, gender, and body mass index (BMI). Tumor size, extension and histopathological diagnosis; with variable outcomes [8]. There were no solid conclusions in published case series to predict the intraoperative and postoperative CSF leakage.

The aim of our study was to present our experience of endoscopic endonasal skull base surgery to identify the predictors associated with intra-and postoperative CSF leaks after an endoscopic endonasal approach (EEA) for resection of pituitary tumors; that could potentially change perioperativ emanagement.

\section{Patients and Methods}

This is a retrospective cohort study conducted at our skull base unit, Neurosurgery Department, Mansoura University Hospitals. Institutional Review Board approval was obtained to perform a retrospective review of patients who underwent endoscopic skull base surgery between June 2015 and December 2018. 150 cases diagnosed with pituitary adenomas were involved in the study. All patients were operated upon via endoscopic transsphenoid approach by the skull base team (neurosurgeons and otolaryngologist). All the patient's medical records were collected. Patient's demographic data, relevant medical information, imaging studies, laboratory studies, anesthetic records, operative records and postoperative data were collected and analyzed. All data regarding previous treatment of the adenomas whether surgical or previous radiation therapy were collected and assessed for its relevance. All patients underwent preoperative radiological evaluation (1.5 tesla MRI brain, sellar views, CT scan of brain and paranasal sinuses with thin slice skull base cuts). Collected MRI finding included; tumor size (maximal tumor diameter), hydrocephalus, pituitary apoplexy, suprasellar extension, associated empty sellae and cavernous sinus invasion. Laboratory data for pituitary function were collected for identification the nature of adenoma. Operative data collected included the technique of endoscopic approach whether mononostril or binostril technique, occurrence of intraoperative CSF leak, the usage of intraoperative lumbar drain and reconstruction methods used for repair. All patients were admitted to ICU overnight of surgery for monitoring and observation. All data of postoperative sequalae were reviewed including CSF rhinorrhea, diabetes insipidus (DI), intracranial infection, and pneumocephalus.

Statistical Analysis

Data was analyzed using (SPSS) Statistical Package for Social Sciences version 20. Categorical variables are presented as absolute number and frequency (\%), and continuous variables are presented as mean \pm standard deviation (SD), range, or median with interquartile range (IQR) intext and tables. All continuous variables were assessed for normality using the Kolmogorov-Smirnov test. If these variables were not normally distributed, descriptive measurements such as median and IQR were calculated. Comparisons of patient characteristics were performed using a Chi-square test or Fisher's exact test for categorical variables in cases with a small number of expected outcomes. All independent variables thought to be of clinical significance were placed into alogistic multiple regression model. A $p$ value of $<0.05$ was considered to indicate a statistically significant difference for all statistical tests.

\section{Results}

A total of 150 endonasal endoscopic transsphenoidal surgeries that met our inclusion criteria. The average age of patients at surgery was $41.82 \pm 5.22$ years ranged from 21 to 65 years. With a relatively equal distribution of females $(n=83,55.4 \%)$ and males $(n=67,44.6 \%)$. Among those 150 cases; 34 cases $(22.6 \%)$ had intraoperative and post-operative CSF leakage. Patients' demographic data, associated medical comorbidities, previous treatment and tumor criteria are summarized in table 1.

Table 1. Perioperative univariate variable analysis of factors affecting incidence of overall CSF leak.

\begin{tabular}{|c|c|c|c|c|}
\hline \multirow{2}{*}{ Variable } & \multicolumn{3}{|c|}{ Mean \pm SD or $(n, \%)$} & \multirow{2}{*}{$P$ value } \\
\hline & All $(n=150)$ & CSF leak $(N=34)$ & No CSF leak $(\mathrm{N}=116)$ & \\
\hline Age (years) & $41.82 \pm 5.22$ & $42.43 \pm 6.51$ & $41.29 \pm 4.48$ & .500 \\
\hline Gende r(male) & $67(44.6 \%)$ & $44 \%$ & $44.8 \%$ & .629 \\
\hline BMI $\left(\mathrm{kg} / \mathrm{m}^{2}\right)$ & $32.4 \pm 1.97$ & $35.5 \pm 2.53$ & $29.56 \pm 1.58$ & $<.001$ \\
\hline Hypertension & $55(36.6 \%)$ & $16(47 \%)$ & $39(33.6 \%)$ & .423 \\
\hline Empty sellae & $2(1.3 \%)$ & $1(2.9 \%)$ & $1(0.8 \%)$ & .168 \\
\hline Preoperative radiation & $4(2.6 \%)$ & $2(5.8 \%)$ & $2(1.6 \%)$ & .001 \\
\hline Recurrence & $16(10.6 \%)$ & $8(23.5 \%)$ & $8(6.8 \%)$ & $<.001$ \\
\hline Pituitary apoplexy & $13(8.6 \%)$ & $4(11.6 \%)$ & $9(7.7 \%)$ & .054 \\
\hline Hydrocephalus & $6(4 \%)$ & $4(11.6 \%)$ & $2(1.6 \%)$ & .001 \\
\hline Mean tumor size & $3.6 \pm 1.2$ & $3.1 \pm 1.14$ & $2.7 \pm 0.9$ & .002 \\
\hline Suprasellar & $40(26.5 \%)$ & $17(50 \%)$ & $23(19.8 \%)$ & $<0.001$ \\
\hline Cavernous sinus & $46(30.6 \%)$ & $13(38.2 \%)$ & $33(28.4 \%)$ & .002 \\
\hline Diabetes insipidus & $6(4 \%)$ & $4(11.7 \%)$ & $2(1.7 \%)$ & .030 \\
\hline Intracranial infection & $3(2 \%)$ & $2(5.8 \%)$ & $1(0.8 \%)$ & .692 \\
\hline
\end{tabular}




\begin{tabular}{lllll}
\hline \multirow{2}{*}{ Variable } & Mean \pm SD or $(\mathbf{n}, \mathbf{\%})$ & & & \\
\cline { 2 - 4 } & All $(\mathbf{n}=\mathbf{1 5 0})$ & CSF leak (N=34) & No CSF leak (N=116) & $<0.001$ \\
\hline Non-Functioning & $90(60 \%)$ & $9(26.4 \%)$ & $81(69.8 \%)$ & \\
Functioning & & & & \\
GH & $28(18.6 \%)$ & $12(35.3 \%)$ & $16(13.7 \%)$ & $<0.001$ \\
PRL & $20(13.4 \%)$ & $9(26.4 \%)$ & $11(9.5 \%)$ & \\
ACTH & $12(8 \%)$ & $8(23.5 \%)$ & $4(3.4 \%)$ & \\
\hline
\end{tabular}

Out of the overall CSF leak; 26 cases (17.3\%) had intraoperative leaks. Out of them; 5 patients $(19.2 \%)$ had postoperative leaks after having repair of an intraoperative leak. 8 patients $(23.5 \%)$ had a postoperative leak without an observed intraoperative leak, 2 of them had delayed leaks more than 30 days after surgery.

Out of 34 cases experienced overall CSF leak; the type of the adenomas was growth hormone-secreting pituitary adenomas $(n=12,35.2 \%)$, prolactin-secreting pituitary adenomas $(n=9,26.4 \%)$, non-functioning pituitary adenomas $(n=9,26.4 \%)$, and adrenocorticotropic hormone-secreting adenomas $(n=8,21.1 \%)$. Radiological analysis of tumors in patients developed CSF leaks revealed: suprasellar extension was reported in 17 pituitary adenomas (50\%), cavernous sinus invasion in 13 patients $(38.2 \%)$, and hydrocephalus in 4 patients $(11.6 \%)$. The mean largest tumor size was $3.1+1.14$ $\mathrm{cm}^{3}$ (Table 1).

Among the 34 cases with overall CSF leakage; 8 cases $(23.5 \%)$ were recurrent adenomas. 6 cases of them (17.6\%) had previous trans-sphenoid surgery only while the other 2 (5.8\%) offered 3-dimensional radiotherapy after the initial trans-sphenoidal surgery. 4 cases $(11.7 \%)$ developed CSF leakage while the surgery was done urgently for pituitary apoplexy.

Univariate analysis of different factors affecting overall leaks revealed: Patients in CSF leak group had mean BMI values $35.5 \pm 2.53 \mathrm{~kg} / \mathrm{m}^{2}$ and patients had not experienced CSF leak had mean BMI values $29.56 \pm 1.58 \mathrm{~kg} / \mathrm{m}^{2},(\mathrm{P}<.001)$. CSF leak rate was $35.3 \%$ in patients with a BMI $>35 \mathrm{~kg} / \mathrm{m}^{2}$, compared with $17.6 \%$ in patients with a BMI $<25 \mathrm{~kg} / \mathrm{m}^{2}$ $(\mathrm{P}=.007)$. Higher rates of CSF leak were found in patients who had suprasellar extension $(50 \%)$ compared to $(19.8 \%)$ in non leak group $(P<.001)$, hydrocephalus $(11.6 \%)$ in leak group vs $(1.6 \%)$ in non-leak group $(P<.001)$, recurrent tumors $(23.5 \%)$ in leak group vs $6.8 \%$ in non-leak group $(\mathrm{P}<.001)$. Urgent surgical intervention for apoplexy needed for 13 patients; 4 patients experienced CSF leak $(11.6 \%)$ of total leak), and 9 patients without leak $(7.7 \%)$ of total nonleak), $(P=.054)$ (table 1$)$.

Patients in the leak group had mean tumor size $3.1 \pm 1.14$ $\mathrm{cm}^{3}$ while patients without leaks had mean maximal tumor diameters $2.7 \pm .91 \mathrm{~cm}^{3}(P=.002)$. The incidence of diabetes insipidus was signifacantly higher in the leak group $(11.7 \%)$ compared to the non leak group $(1.7 \%)(p=.030)$. Multiple logistic regression model analysis (Table 2) revealed that BMI, recurrent cases, hydrocephalus, and suprasellar extension, were statistically significance in relation to incidence of overall CSF leak.
Table 2. Multiple logistic regression model analysis of factors affecting incidence of CSF leaks.

\begin{tabular}{lcll}
\hline CSF leak & $\mathbf{9 5 \%} \mathbf{C I}$ & \multicolumn{1}{l}{ Odds Ratio } & P value \\
\hline Overall: & & & \\
BMI & $1.03-1.09$ & 1.05 & 0.017 \\
Suprasellar & $1.36-3.41$ & 2.10 & 0.006 \\
Hydrocephalus & $1.41-5.23$ & 2.53 & 0.007 \\
Recurrence & $1.02-2.21$ & 3.21 & 0.041 \\
Intraoperative: & & & \\
BMI & $1.03-1.09$ & 1.05 & 0.011 \\
Suprasellar & $1.49-3.98$ & 2.48 & $<0.001$ \\
Hydrocephalus & $1.56-5.78$ & 3.10 & 0.003 \\
Postoperative: & & & \\
BMI & $1.02-1.11$ & 1.03 & 0.033 \\
Hydrocephalus & $1.13-9.8$ & 3.89 & 0.010 \\
\hline
\end{tabular}

Univariate analysis of different factors affecting intraoperative leaks revealed (table 3): No statistical significance found between leak and non-leak groups as regard previous skull base radiation, pituitary apoplexy, and cavernous sinus invasion. Patients who had mean BMI values $33.5+2.43$ had a significant incidence of CSF leak compared to patients had $30.12+2.11 \mathrm{~kg} / \mathrm{m}^{2},(P<0.001)$. Higher rates of intraoperative CSF leak were found in patients who had suprasellar extension, hydrocephalus, and recurrent tumors. Multiple logistic regression model analysis (Table 2) revealed that BMI, hydrocephalus, and suprasellar extension, were significantly correlated with incidence of intraoperative CSF leaks.

Table 3. Perioperative univariate variable analysis offactors affecting incidence of intraoperative CSF leak.

\begin{tabular}{llll}
\hline \multirow{2}{*}{ Variable } & \multicolumn{2}{c}{ Mean \pm SD or (n,\%) } & \multirow{2}{*}{ P value } \\
\cline { 2 - 3 } & $\begin{array}{l}\text { Intraoperative } \\
\text { CSF leak (n=26) }\end{array}$ & $\begin{array}{c}\text { No CSF leak } \\
(\mathbf{n = 1 2 4})\end{array}$ & \\
\hline Age (years) & $44.15 \pm 7.34$ & $43.95 \pm 6.23$ & .642 \\
Gender (male) & $15,57.6 \%$ & $52,41.2 \%$ & .261 \\
BMI $\left(\mathrm{kg} / \mathrm{m}^{2}\right)$ & $33.5 \pm 2.43$ & $30.12 \pm 2.11$ & $<.001$ \\
Hypertension & $13,50 \%$ & $32,25.8 \%$ & .053 \\
Empty sellae & 0 & $2,1.6 \%$ & .065 \\
Preoperative radiation & $1,3.8 \%$ & $3,2.4 \%$ & .421 \\
Recurrence & $7,26.9 \%$ & $9,7.2 \%$ & $<.001$ \\
Pituitary apoplexy & $3,11.5 \%$ & $10,8.1 \%$ & .063 \\
Hydrocephalus & $3,11.5 \%$ & $3,2.4 \%$ & $<.001$ \\
Max tumor size & $2.9 \pm 1.1$ & $2.02 \pm .83$ & .002 \\
Suprasellar & $16,61.5 \%$ & $24,19.3 \%$ & $<.001$ \\
Cavernous sinus & $17,65.3 \%$ & $29,23.3 \%$ & .002 \\
Non-Functioning & $15,57.7 \%$ & $75,60.5 \%$ & .501 \\
Functioning & & & \\
GH & $8,30.7 \%$ & $20,16.1 \%$ & \\
PRL & $7,26.9 \%$ & $13,10.4 \%$ & .002 \\
ACTH & $5,19.2 \%$ & $7,5.6 \%$ & \\
\hline
\end{tabular}


Univariate analysis of different factors affecting postoperative CSF leak revealed (Table 4). No statistical significance found between leak and non-leak groups as regard previous skull base radiation, pituitary apoplexy, and cavernous sinus invasion.

Mean BMI values for patients with CSF leak was $34.5 \pm 5.3$ while mean $\mathrm{BMI}$ values for patients in non-leak group was $30.12 \pm 3.62(P=.003)$. Postoperative CSF leak was observed in older patients (mean age, $49.6 \pm 14.3$ vs $41.5 \pm 11.7$ years; $P=.038$ ). Multiple logistic regression model analysis (Table 2) revealed that, BMI and hydrocephalus were significantly correlated with occurrence of postoperative CSF leaks.

Table 4. Perioperative univariate variabl eanalysis of factors affecting incidence of postoperative CSF leak.

\begin{tabular}{llll}
\hline \multirow{2}{*}{ Variable } & \multicolumn{2}{l}{ Mean \pm SD or (n, \%) } & \\
\cline { 2 - 3 } & $\begin{array}{l}\text { Postoperative } \\
\text { CSF leak (n=13) }\end{array}$ & $\begin{array}{l}\text { No CSF leak } \\
\text { (n=137) }\end{array}$ & P value \\
\hline Age (years). & $49.6 \pm 14.3$ & $41.5 \pm 11.7$ & .038 \\
Gender (male) & $7,53.8 \%$ & $60,43.7 \%$ & .687 \\
BMI $\left(\mathrm{kg} / \mathrm{m}^{2}\right)$ & $34.5 \pm 5.3$ & $30.12 \pm 3.62$ & .003 \\
Hypertension & $8,61.5 \%$ & $47,34.3 \%$ & .052 \\
Empty sellae & $1,7.6 \%$ & $1,0.7 \%$ & $<.001$ \\
Preoperative radiation & $1,7.6 \%$ & $3,2.1 \%$ & .420 \\
Recurrence & $9,69.2 \%$ & $7,5.1 \%$ & $<.001$ \\
Pituitary apoplexy & $2,15.2 \%$ & $11,8 \%$ & 1.0 \\
Hydrocephalus & $4,30.4 \%$ & $2,1.4 \%$ & $<.001$ \\
Maximum tumor size & $3.5 \pm 1.3$ & $2.9 \pm 1.1$ & .019 \\
Suprasellar & $8,61.5 \%$ & $32,23.3 \%$ & $<.016$ \\
Cavernous sinus & $2,15.2 \%$ & $44,32.1 \%$ & .002 \\
Non-Functioning & $6,45.6 \%$ & $84,61.3 \%$ & .564 \\
Functioning & & & \\
GH & $3,23.1 \%$ & $25,18.2 \%$ & \\
PRL & $2,15.2 \%$ & $18,13.1 \%$ & .012 \\
ACTH & $2,15.2 \%$ & $10,7.2 \%$ & \\
\hline
\end{tabular}

Higher postoperative leak rate occurred in patients underwent septal flap repairs in comparison to other repair techniques (54\% vs 30.7, 15.3\%, P=.063) (table 5). Only 3 patients with intraoperative leak and 8 patients with postoperative leak were managed by using lumbar drains.

Table 5. Methods of sellar reconstruction for intraoperative CSF leaks and the rates of resulted postoperative leak.

\begin{tabular}{lc}
\hline Reconstruction technique & Postoperative CSF leak= 13 (n, \%) \\
\hline Septal flap & $7,54 \%$ \\
Middle turbinate flap & $4,30.7 \%$ \\
Multilayer reconstruction & $2,15.3 \%$ \\
\hline
\end{tabular}

\section{Discussion}

CSF leak is one of the most common complications associating trans-sphenoid approach for removing pituitary adenomas [3]. Despite its well-known and evident prevalence, there are limited research studies investigating the risk factors associated with increased rate of CSF rhinorrhea and its clinical consequences [16].

Endoscopic skull base surgeons treating patients with pituitary adenomas can potentially change or modify their clinical management strategy to minimize the incidence of CSF leakage and its subsequent morbidities [17]. Prediction of the possibility for intraoperative or postoperative CSF leakage in endoscopic endonasal surgery for pituitary adenomas is of great interest and is discussed in some the recent publications $[5,18]$.

Identification of such risk factors enables the surgeon to plan initially to avoid intra-operative CSF leakage and to prepare for how to deal with such complication if occurred [13]. Ourcurrent study reported an overall incidence of CSF leakage in $22.6 \%$ of cases operated upon for pituitary tumors using the endoscopic trans-sphenoid approach.

The incidence of postoperative CSF leakage in association with endoscopic trans-sphenoid approach for pituitary adenomas ranged between 1.4 and $16.9 \%$ in many series [1, 17], our rate of postoperative CSF leak was $8.6 \%$. Although statistically insignificant; we had a slight female predominance in the incidence of CSF leakage which is same in some other studies that reported high incidence of CSF leakage in females [17].

There is a significant association between elevated BMI and both intraoperative and postoperative CSF leakage [17, 19]. Theories suggest that increase in pleural pressure and cardiac filling pressure may impede the brain venous return which cause increase in the intracranial venous pressure and hence increase the ICP. This pathophysiology is widely accepted in the pathogenesis of benign intracranial hypertension and empty sellae syndrome [20].

Ivan et al. found that increased BMI was associated with a higher incidence of postoperative CSF leakage in a cohort of 98 consecutive patients with expanded endoscopic transsphenoid approach performed at the University of California [13]. In current study of 150 patients, overall CSF leak was in 34 cases $(22.6 \%)$; there was a statistically significant association between elevated BMI and both intraoperative and postoperative CSF leak $(\mathrm{p}<0.001)$.

Although relatively uncommon, intraventricular extension had the greatest association with a postoperative CSF leak in many studies $[13,17]$. This association is likely due to the increased risk of a high flow leak. However, in this study, 6 cases with intraventricular extension have been encountered. In addition, suprasellar extension had an interesting impact on the CSF leak especially the intraoperative in many studies $[12,21]$. In our study we encountered statistically significant association between suprasellar tumor extension and the risk of CSF leak $(\mathrm{p}<.001)$.

This study copes with the literature in the identified risk factors like the high BMI, the presence of suprasellar tumor extension, the operated recurrent cases, the irradiated cases, the presence of intraoperative CSF leak [1, 13, 19, 20].

Regarding prior treatment; Nishioka et al. found that previous trans-sphenoidal surgery and skull base radiation were each in dependent risk factor for occurrence of postoperative CSF rhinorrhea [12]. Among our total 150 cases; there were 16 cases of recurrent adenomas (10.6\%). Out of recurrent cases; 8 patients developed CSF leak (50\%). While, 134 denovo cases only 26 cases (18.1\%) developed CSF leak. In our study; we found statistical significance between recurrence and previous radiotherapy with the risk 
of postoperative CSF leakage $(\mathrm{p}<.001)$.

The routine usage of lumbar drain with endoscopic transsphenoid approach is a matter of great debate and there is still some debate about its value with occurrence of intraoperative and post-operative CSF leakage $[14,22]$. Many studied had underestimated its benefit in management of CSF rhinorrhea associating endoscopic trans-sphenoid surgery for pituitary adenomas [23]. Other studies considered the pattern of CSF leakage and recommended not to use lumbar drains for low-flow leakage and insert lumbar drain for high flow leakage with known or suspected intracranial hypertension or high possibilities for reconstruction failure [23]. In this study the authors limited the use of lumbar drain for cases of high flow CSF leak. However, in cases of low flow CSF leak lumbar drain was not performed. Lumbar drain was inserted in 8 patients of the postoperative CSF leak group and in 3 patients of the intraoperative CSF non-leak group.

Many reconstruction methods for the sella were advocated over the past few years $[9,11]$. Many studies depend on fat or fascia lata for reconstruction while others use the previously described Hadad nasoseptal flap [10, 14, 22]. Intraoperative management of CSF leak depend on immediates urgical decision-making, which depend on factors such as surgery extensiveness, surgeon experience, specialty training (neurosurgery vs. otolaryngology) [3]. In this study the authors had the opinion that, when low-flow CSF leak was inspected, anautologous fat or fascial at a graft was placed with in the dural defect as an inlay graft, bone chips from the posterior septum were used as onlay graft. The authors covered all this with posterior septal flap. On the situation that high-flow CSF leak occurred, combined use of fat and facia lata and or a vascularized pedicled nasoseptal flap was harvested from nasal septum and covered on the overlay graft.

Some studies had investigated the type of adenoma as an impact factor for postoperative CSF leak $[17,18]$. However, the type of adenoma has not affected the rate of CSF leak in a statistically significant [17]. In this study, we noticed a higher incidence of postoperative CSF leak in cases of acromegaly and Cushing type of adenoma; one case associated with empty sella syndrome. This difference from the literature can be explained by the great anatomical variation of the skull base in these two types of adenomas [4, 21].

\section{Conclusion}

Prediction of CSF leak and good identification of risk factors are of great importance in endoscopic transsphenoidal surgery for pituitary adenoma. Elevated BMI, suprasellar extension of the tumor, recurrence or previous radiation and special types of adenoma are all important risk factors that have been proved in this study to have statistically significant association with the incidence of CSF leak. In high risk patients for CSF leakage; the surgical team should prepare a good and precise reconstruction plan to deal with occurrence of CS Fleak.

\section{References}

[1] S. K. Rereddy, Z. M. Patel, N. M. Oyesiku, et al. "Factors impacting cerebrospinal fluid leak rates in endoscopic sellar surgery". Int Forum Allergy Rhino 10 (0) :1-9, 2016.

[2] Z. M. Soler, W. A. Vandergrift, S. K. Wiseetal." Transnasal endoscopic pituitary adenoma surgery". Int Forum Allergy Rhino 16 (1): 101-7, 2016.

[3] C. J. Farrell, A. Farag, J. J. Evansetal." Principles of Pituitary Surgery". Otolaryngol Clin North Am 13 (1), 2018.

[4] E. C. Kuan, A. R. Sepahdari, F. Yooetal." Anatomic Variations in Pituitary Endocrinopathies: Implications for the Surgical Corridor". J Neurol Surg B Skull Base 105-11, 2017.

[5] A. Parikh, S. E. Sullivan, E. L. Mckean." Predictive Factors, 30-Day Clinical Outcomes, and Costs Associated with Cerebrospinal Fluid Leak in Pituitary Adenoma Resection". J Neurol Surg 103-111, 2019.

[6] C. Zhang, N. Yang, L. Muetal." The application of nasoseptal" rescue" flap technique in endoscopic transsphenoidal pituitary adenoma resection". Acta Otorhinolaryngol Ital 37: 303-307, 2018.

[7] A. Chern, J. B. Hunter, M. L. Bennett." Cost analysis of cerebrospinal fluid leaks and cerebrospinal fluid leak prevention in patients undergoing cerebellopontine angle surgery". Otol Neurotol 38 (01): 147-151, 2017.

[8] C. C. Boling, T. T. Karnezis, A. B. Baker et al." Multiinstitutional study of risk factors for perioperative morbidity following transnasal endoscopic pituitary adenoma surgery". Int Forum Allergy Rhinol 6 (01): 101-107, 2016.

[9] J. Barger, M. Siow, M. Kader et al." The posterior nasoseptal flap: A novel technique for closure after endoscopic transsphenoidal resection of pituitary adenomas". Surg Neurol Int 9: 32, 2018.

[10] J. Park, J. H. Choi, Y. Kim et al." Modified Graded Repair of Cerebrospinal Fluid Leaks in Endoscopic Endonasal Transsphenoidal Surgery". J Korean Neurosurg Soc 58 (1): 36-42, 2015.

[11] P. Schuss. "Transsphenoidal pituitary surgery: comparison of two sellar reconstruction techniques and their effect on postoperative cerebrospinal fluid leakage". Neurosurg Rev 41 (4): 1053-1058, 2018.

[12] H. Nishioka, J. Haraoka, Y. Ikeda. "Risk factors of cerebrospinal fluid rhinorrhea following transsphenoidal surgery". Acta Neurochir 147 (11): 1163-1166, 2005.

[13] M. E. Ivan, J. B. Iorgulescu, I. El-Sayed et al." Risk factors for postoperative cerebrospinal fluid leak and meningitis after expanded endoscopic endonasal surgery". J Clin Neurosci 22 (1): 48-54, 2015.

[14] A. J. Luginbuhl, P. G. Campbell, J. Evans et al." Endoscopic Repair of High-Flow Cranial Base Defects Using a Bilayer Button". Laryngoscope 876-80, 2010.

[15] S. Fraser, P. A. Gardner, M. Koutourousiou et al." Risk factors associated with postoperative cerebrospinal fluid leak after endoscopic endonasal skull base surgery". J Neurosurg 5-10, 2017. 
[16] D. Jakimovski, G. Bonci, M. Attia et al." Incidence and significance of intraoperative cerebrospinal fluid leak in endoscopic pituitary surgery using intrathecal fluorescein". World Neurosurg 82 (3-4): e513-e523, 2014.

[17] N. R. Biermasz and W. R Furth." Preoperative risk factors for postoperative complications in endoscopic pituitary surgery: a systematic review Quality in Prognostic Studies". Pituitary 21 (1): 84-97, 2018.

[18] P. N. Patel, A. M. Stafford, J. R Patrinely et al." Risk Factors for Intraoperative and Postoperative Cerebrospinal Fluid Leaks in Endoscopic Transsphenoidal Sellar Surgery". Otolaryngol Head Neck Surg 158 (5): 952-960, 2018.

[19] I. Sun, J. X. Lim, C. P. Goh et al." Body mass index and the risk of postoperative cerebrospinal fluid leak following transsphenoidal surgery in an Asian population". Singapore Med J 59 (05): 257-263, 2018.
[20] B. J. Dlouhy, K. Madhavan, J. D. Clinger et al." Elevated body mass index and risk of postoperative CSF leak following transsphenoidal surgery". J Neurosurg 116 (06): 1311-1317, 2012.

[21] G. Carrabba, M. Locatelli, L. Mattei et al." Transphenoidal surgery in acromegalic patients: Anatomical considerations and potential pitfalls". Acta Neurochir 155: 125-130, 2012.

[22] R. Zhan, A. S. Chen, A. S. Xu et al." Postoperative Low-Flow Cerebrospinal Fluid Leak of Endoscopic Endonasal Transsphenoidal Surgery for Pituitary Adenoma. Wait and See or Lumbar Drain"? J Craniofac Surg 26 (4): 1261-4, 2015.

[23] D. A. Tien, J. K. Stokken, P. F. Recinos et al." Cerebrospinal Fluid Diversion in Endoscopic Skull Base Reconstruction An Evidence-Based Approach to the Use of Lumbar Drains". Otolaryngol Clin NA 49 (1): 119-29, 2016. 